Please do not remove this page

RMIT

UNIVERSITY

\title{
Observation of a smecticlike crystalline structure in polydisperse colloids
}

Martin, Stephen; Bryant, Gary; Van Megen, William

https://researchrepository.rmit.edu.au/esploro/outputs/9921858635701341/filesAndLinks?institution=61 RMIT_INST\&index=null

Martin, S., Bryant, G., \& Van Megen, W. (2003). Observation of a smecticlike crystalline structure in polydisperse colloids. Physical Review Letters, 90(25), 255702-1-255702-255704.

https://doi.org/10.1103/PhysRevLett.90.255702

Document Version: Accepted Manuscript

Published Version: https://doi.org/10.1103/PhysRevLett.90.255702

Repository homepage: https://researchrepository.rmit.edu.au

(c) 2003 The American Physical Society

Downloaded On 2023/04/27 01:25:45 +1000 
Thank you for downloading this document from the RMIT Research Repository.

The RMIT Research Repository is an open access database showcasing the research outputs of RMIT University researchers.

RMIT Research Repository: http://researchbank.rmit.edu.au/

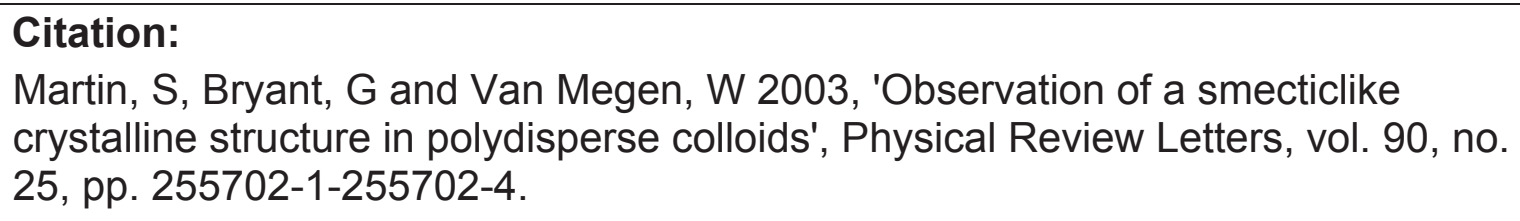

See this record in the RMIT Research Repository at:

https://researchbank.rmit.edu.au/view/rmit:3791

Version: Accepted Manuscript

\section{Copyright Statement:}

(C) 2003 The American Physical Society

Link to Published Version:

http://dx.doi.org/10.1103/PhysRevLett.90.255702 
Please cite as:

Martin, S., Bryant, G., Van Megen, W.

"Observation of a smecticlike crystalline structure in polydisperse colloids," Physical Review Letters 90, 25570212557024 (2003). 


\title{
Observation of a smectic-like crystalline structure in polydisperse colloids.
}

\section{Stephen Martin, Gary Bryant* and William van Megen}

Department of Applied Physics, Royal Melbourne Institute of Technology, GPO Box, 2476V, Melbourne 3001, Australia

*gary.bryant@rmit.edu.au

\begin{abstract}
We present the results of crystallographic measurements on samples of two latexes, one with a relatively symmetric particle size distribution, and another with a highly skewed pseudo-bimodal distribution. For the skewed latex, crystallites are clearly visible, but they exhibit only a single Bragg reflection, indicating long-range order in only one direction. We propose a schematic model that explains this result in terms of stacks of planes, which are unregistered due to a high incidence of stacking faults caused by the incorporation of a large number of small particles.
\end{abstract}

PACS Number(s): 64.70.Dv, 81.10.Fq, 82.70.Dd 
It is well known that at volume fractions beyond 0.494 , single component hard spheres crystallize into close-packed hexagonal layers. Such stacking can take the form of Face-Centered Cubic FCC (an ABCABC stacking sequence), Hexagonal Close Packed HCP (an ABAB stacking sequence), or random hexagonal close packed (RHCP), a mixture of the two where there is random registration of planes.

Experimentally it is found that hard sphere colloids which crystallize quickly from the melt exhibit RHCP stacking [1,2], while FCC-like stacking can be induced through the application of shear [3]. More complicated systems, such as binary mixtures, can exhibit superlattice formation [4].

However, colloidal particles are inherently polydisperse. Models of hard-sphere systems show that, even for small polydispersities $(<10 \%)$, there is local partitioning or segregation of particle sizes [5]. Other studies [6] show that any partitioning in a sample, for example via eutectic or compound formation, has a strong retarding effect on nucleation. However in a single component system there is no evidence that the crystal is anything other than a random hexagonal close-packed (RHCP) structure.

In this paper we report qualitatively different crystal structures for two suspensions of polymer particles (identified as samples $\mathrm{X}$ and $\mathrm{W}$ ) with different particle size distributions. One of the suspensions exhibits hexagonal close packing with random registration of planes (RHCP), while the other exhibits packing with no registration of planes, comparable with the smectic phase of a liquid crystal.

Both samples are composed of hard sphere-like polymer particles suspended in cisdecalin for refractive index matching. These particles behave as near perfect hard 
spheres as shown from phase diagrams [7] and direct measurements [8]. The two samples have qualitative, although quantifiable differences in their Particle Size Distributions (PSDs), but have equilibrium phase diagrams which are similar, within the normal errors associated with these measurements.

The PSDs are shown in figure 1. These were determined from light scattering measurements of the angular dependence of the intensity and apparent radius. These properties are very sensitive to the PSD when traversing intensity form factor minima, allowing for accurate determinations of the PSD [9]. Latex X has a slightly negatively skewed size distribution of particles, with a polydispersity of approximately $7 \%$. For latex W, the spectroscopic data cannot be explained by a uni-modal PSD - in this case a continuous bimodal distribution provides the best agreement with the spectroscopic data, and is consistent with other measurements (see [9] for details). The distribution of the smaller component is so broad that the PSD can practically be considered a single distribution with an extreme skewness.

Results presented here are for sample volume fractions of $\phi=0.54$. Prior to each measurement the samples were tumbled to shear melt any crystals and then left undisturbed. All results described here apply to homogeneously nucleated crystals after the completion of spontaneous crystallization and growth (1 day for X, 3 days for W). A detailed analysis of the kinetics of crystal growth has been presented elsewhere [10].

Photographs of suspensions $\mathrm{X}$ and $\mathrm{W}$ shown in figures $2 \mathrm{a}$ and $2 \mathrm{~b}$ are typical of colloidal crystals of hard sphere particles [11]. The crystals are small, and in order to 
obtain good averaging, scattered laser light is collected from an area of $\sim 1 \mathrm{~cm}^{3}$, analogous to X-ray powder crystallography [1]. In this work the averaging over crystal orientations is improved further by rotating the detector over the DebyeScherrer cone [12].

Figure 3 shows the scattered intensity for the two samples over the range of scattering vectors accessible to our experiment. For sample $\mathrm{X}$ there are six reflections (indicated by the bold vertical lines) observed over the accessible spatial range. These peaks are invariant to the position of the sample with respect to the laser beam and the position of the detector on the Debye-Scherrer cone. Calculation of the ratios of the observed peaks in sample X shows the structure to be RHCP, with the main interlayer reflection occurring at $q R=3.5$.

By contrast, sample $\mathrm{W}$ has only one reproducible reflection at $q R=3.64$. The other small features at high $q R$ are not reproducible - they depend on the position of the sample and the position of the detector on the Debye-Scherrer cone. The presence of the single reflection in sample $\mathrm{W}$ indicates a solid phase ordered in one direction, but with very little correlation in perpendicular directions. Sample W crystallites therefore consist of an unregistered stacking of planes. These qualitative differences in the crystal structure of samples $\mathrm{X}$ and $\mathrm{W}$ are also found at $\phi=0.55$ and $\phi=0.53$, and are completely reproducible. For volume fractions $\phi \geq 0.56$ sample $\mathrm{W}$ formed a glass.

To further examine this behaviour, the effects of applied shear forces were studied. It is well known that the application of shear to colloidal solids has a significant effect 
on the rate and type of crystallization [e.g. 13-16]. For crystalline samples shear can drive RHCP crystals towards the FCC structure [3, 15]. In addition, shearing can induce crystallization in samples which are too polydisperse or too concentrated to crystallize under when left completely undisturbed [16].

To investigate these effects, both samples were oscillated at $1 \mathrm{~Hz}$ for times comparable with the crystallization times when left undisturbed [see 17]. Figures 2(c) and 2(d) show photographs of samples $\mathrm{X}$ and $\mathrm{W}$ respectively, immediately after sample oscillation had ceased. Sample X behaves as expected, with nothing obviously different between 2(a) and 2(c). Measurement of the scattered intensity for the sheared sample X (data not shown) indicates suppression of the HCP (100) and (101) reflections and the emergence of an FCC (200) peak, similar to the results of Kegel and Dhont [15]. For sample $\mathrm{X}$ the oscillations guide the sample closer to equilibrium, leading to a more FCC-like structure.

Sample W, however, shows quite different behaviour. Figure 2(d) shows a sheared region (within the dotted line) in which there appear to be fewer crystallites, surrounded by an apparently unchanged nucleated region. Several repeats of the experiment gave the same result. Figure 4 shows the scattering intensity from the different regions of the sample. With both the nucleated and sheared region illuminated simultaneously, the reflection is shown in line 2. This reflection appears to be a superposition of a peak from the nucleated region, at approximately the same scattering vector as when the sample was not oscillated (line 1), and a separate peak from the sheared region positioned at a lower scattering vector. To confirm this, lines (3) and (4) show the scattered intensities from the nucleated and sheared regions, 
when these regions were illuminated separately. The annealing produced by the oscillation results in crystallites in which the distance between reflecting layers increases, but there is still no evidence of registration of planes.

Latex $\mathrm{W}$ can be considered to be a (continuous) bimodal distribution with a size ratio of about 0.57 . The equilibrium crystal structure for hard spheres with such a complex distribution has not been considered. The nearest approximation is that of a binary hard sphere system with a size ration of 0.57 , where superlattice structures, such as $\mathrm{AB}_{2}$ and $\mathrm{AB}_{13}$ are expected to occur [e.g. 18].

It is possible that some of the smaller particles within the distribution are of a size that can slip into interstices within the main lattice. However, as the smaller particles themselves have a broad size distribution, such interstitial particles are more likely to occur randomly as interstitial point defects, rather than forming a (regular) sub-lattice. As planes of the average (larger) sized particles form, these smaller particles could become trapped between and within the planes. These spatially random substitutions of small particles both destroy long range intra-plane (spatial) correlation, and increase the average intra-plane spacing of particles. So the only source of coherence comes from the interplanar spacing.

A simple schematic of this proposed structure for sample $\mathrm{W}$ crystallites is shown in figure 5. The upper figure shows a cross section of a regular hexagonal crystal, set at an arbitrary interlayer distance of 1 . The lower figure shows the proposed stacking of the $\mathrm{W}$ crystallites. The intra-plane spacing between particles in the top and bottom layers has been increased, and the spacing between the layers has been reduced to 
about 0.8 , which conserves the particle density. This conservation of the density has been assumed as we expect the crystallites of sample $\mathrm{W}$ to have a similar packing fraction to those in sample $\mathrm{X}$. The change in the spacing of the layer results as a consequence of particles much smaller than the average size being incorporated into the crystallites as point defects, disrupting the formation of a regular hexagonal stacking sequence. The smaller particles remain either due to kinetic constraints, or because of a drive to locally create a superlattice structure. It can also be seen in figure 5 how the planes now become unregistered; while the three planes shown are on average parallel, particles in the vertical direction no longer line up. This deregistering of the planes is more severe when the schematic is extended to three dimensions.

The application of shear on the sample fractionates out some of the defect particles from the crystallites. The slight reduction in polydispersity within the crystallites causes less disruption to the particle spacing in the planes, and the crystal formed in the sheared region of figure $2 \mathrm{~d}$ tends towards a regular stacking of hexagonal layers. This in turn allows the planes to sit further away from each other.

We have found evidence for an intermediate smectic-like structure present in the presence of a highly skewed (or continuous bimodal) particle size distribution. We speculate that the structure of this phase is one in which a large number of point defects occur within the crystallites, which locally may replicate complex superlattice structures, but which produce no long range order within layers. This observation suggests that the effects of polydispersity in hard-spheres may be more subtle than is generally realized. 
1. $\quad$ P.N. Pusey et al., Phys. Rev. Lett., 63, 2753 (1989).

2. $\quad$ P.N. Pusey, in Liquids, Freezing and the Glass Transition, edited by J.P.

Hansen, D. Levesque, and J. Zinn-Justin (North Holland, Amsterdam. 1991) p. 763;

P. Bartlett and W. van Megen, in Granular Matter. (Springer-Verlag, New York, 1994) p. 195.

3. An excellent recent paper which reviews much of this material is: V.C.

Martelozzo, A.B. Schofield, W.C.K. Poon and P.N. Pusey, Phys. Rev. E, 66, 0210408 (2002).

4. P. Bartlett, R.H. Ottewill and P.N. Pusey, J. Chem. Phys. 93, 1299 (1990);

P. Bartlett, R.H. Ottewill and P.N. Pusey, Phys. Rev. Lett. 68, 3801 (1992).

5. D.A. Kofke and P.G. Bolhuis, Phys. Rev. E 59, 618 (1999).

6. S. Henderson, $\mathrm{PhD}$, thesis, Royal Melbourne Institute of Technology, 1999;P. Bartlett, J. Phys.: Cond. Matt. 12, A275 (2000); R.M.L. Evans and C.B. Holmes, Phys. Rev. E 64, 1404 (2001).

7. S.M. Underwood and W. van Megen, Coll. Polymer Sci. 274, 1072 (1996).

8. G. Bryant et al., Phys. Rev. E 66, 060501 (R) (2002).

9. G. Bryant, S. Martin, B. Budi and W. van Megen, Langmuir 19, 616 (2003).

10. S. Martin, G. Bryant and W. van Megen, Phys. Rev. E (in press).

11. P.N. Pusey and W. van Megen, Nature (London) 320, 340 (1986).

12. P. Francis P. et al., Rev. Sci. Instrum. 73, 3872 (2002).

13. B.J. Ackerson, J. Rheol. 34, 553 (1990).

14. J. Zhu et al., Nature (London) 387, 88 (1997).

15. W.K. Kegel and J.K.G. Dhont, J. Chem. Phys. 112, 3431 (2000).

16. W. van Megen and S.M. Underwood, Nature (London) 362, 616 (1993); S.I.

Henderson, T.C. Mortensen, S.M. Underwood and W. van Megen, Physica A 233, 102 (1996); M.D. Haw, W.C.K. Poon and P.N. Pusey, Phys. Rev. E, 57, 6859 (1998).

17. S. Martin, PhD, thesis, Royal Melbourne Institute of Technology, 2002.

18. M.J. Murray and J.V. Sanders, Phil. Mag. A 42, 721 (1980); P. Bartlett, J. Phys. Condens. Matter 2, 4979 (1990). 
FIG. 1. Particle size distributions of the radius for samples $X$ (dashed line) and $\mathrm{W}$ (solid line) determined from fits to the static and dynamic light scattering measurements made on dilute suspension [8]. For sample W there is a significant fraction of particles continuously distributed at sizes much smaller than the average radius (approximately $18 \%$ of particles have a radius below $214 \mathrm{~nm}$ ). The distribution is a continuous bimodal with peaks at $245 \mathrm{~nm}$ and $140 \mathrm{~nm}$ (the latter is indicated by an arrow).

FIG. 2. Photographs of samples $X(a)$ and $W(b)$ at a volume fraction of 0.54 , taken three days after shear melting. The scale divisions are in millimeters. Figures (c) and (d) show the same two samples immediately following sample oscillation at $\approx 1 \mathrm{~Hz}(1$ day for $X$ and 2 days for $W$ ). Sample $X$ shows uniform crystallite coverage throughout the sample. Sample W shows a large, more uniform central region (dotted line), surrounded by regular crystallites.

FIG. 3. Scattered intensity over the accessible range for sample $X$ (upper line) and sample W (lower line) both at $\phi=0.54$. The vertical lines show possible Bragg reflections, labeled with both $\mathrm{HCP}$ and FCC notation, assuming the primary reflection for sample $\mathrm{X}$ is due to the distance between close packed planes of an RHCP crystal. FIG. 4. Scattered intensity of sample W when left to stand undisturbed (line (1)), and after two days of oscillation following the cessation of shear melting (line (2)). Line (2) appears to be two peaks superimposed onto each other. To confirm this, the incident laser beam was shaped using a rectangular aperture so that the nucleated and sheared regions were illuminated separately. The results are shown as lines (3) and (4). The fluid structure factor is also shown with line (4) for comparison.

FIG. 5. Schematic of the proposed local structure of a sample W crystallite. The upper diagram shows a regular hexagonal stacking where the space between three layers is defined in arbitrary units as 1 . In the lower diagram some smaller particles have become positioned within the lattice. As a consequence particles within the parallel layers are spread out further in the horizontal direction (relative to the regular stacking sequence shown above), and the planes are now closer together. Although the spacing between planes is closer, spreading of the particles within the planes conserves the density, so the lower diagram is approximately the same density as the upper diagram. 


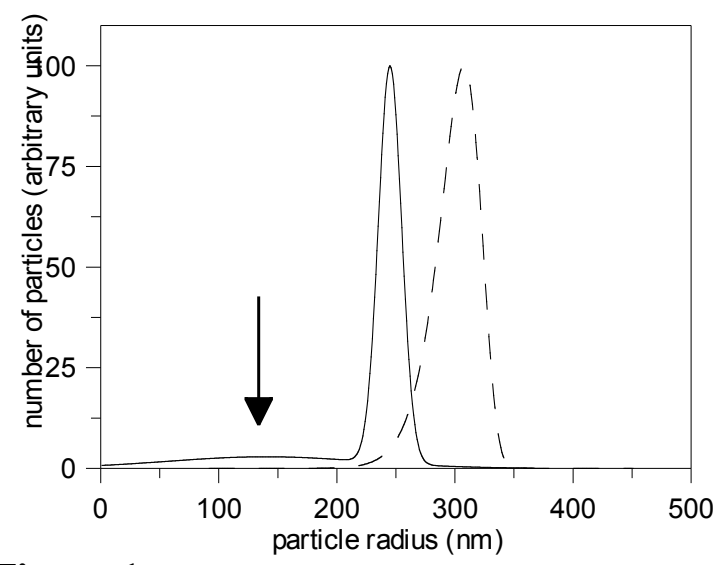

Figure 1. 


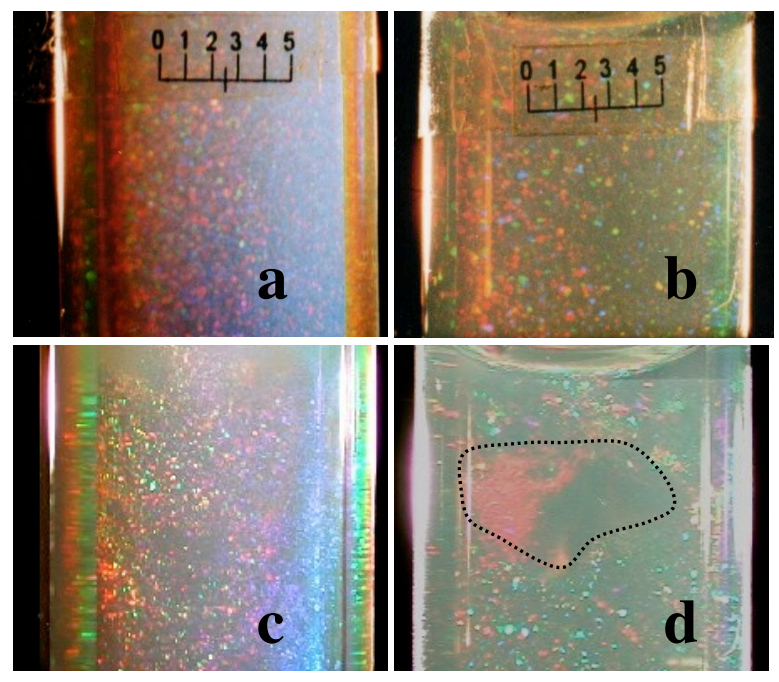

Figure 2. 


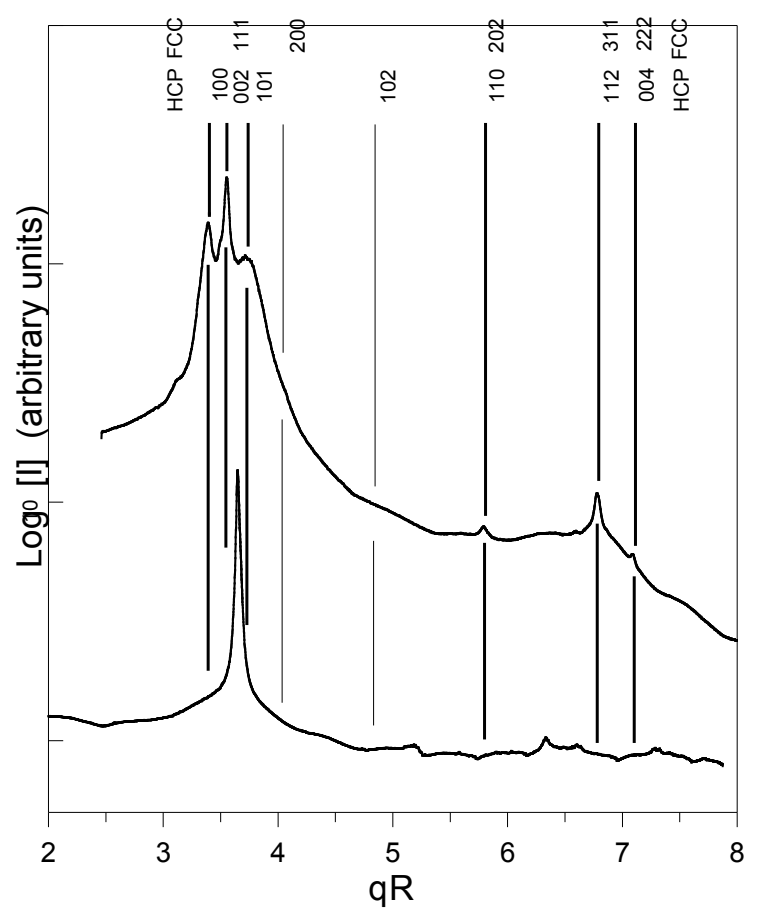

Figure 3. 


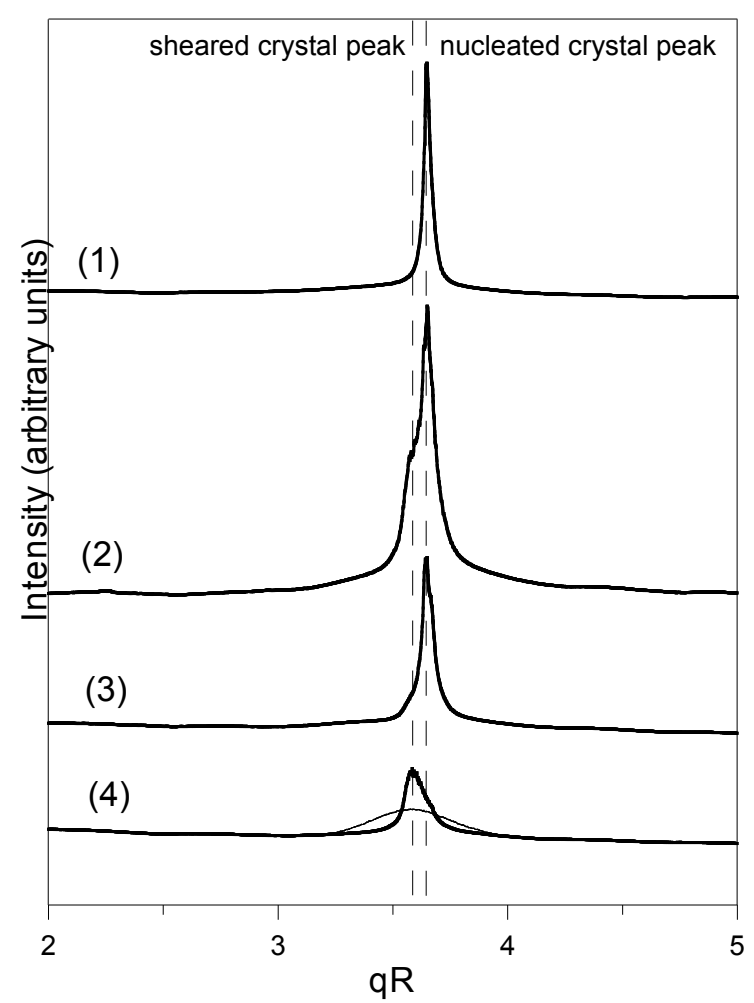

Figure 4.

13 


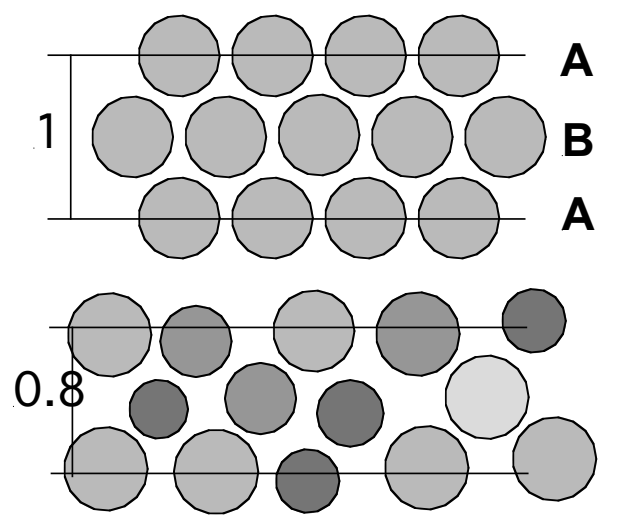

Figure 5. 